УДК 537.87

DOI: 10.31471/1993-9981-2018-2(41)-100-112

\title{
НОВИЙ РОЗВ'ЯЗОК КАНОНІЧНОЇ ЗАДАЧІ РОЗСІЮВАННЯ ХВИЛЕВОДНИХ МОД НА КРУГОВОМУ ПРОВІДНОМУ ЦИЛІНДРІ
}

\author{
А. В. Семенчук ${ }^{1 *}$, В. В. Петрусенко ${ }^{2}$, Я. В. Чумаченко \\ ${ }^{1}$ Івано-Франківський національний технічний університет нафти і газу, \\ вул. Карпатська, 15, м. Івано-Франківськ, Украӥна, 76019, е-таil:andrijsemenvhuk@gmail.com, \\ tel. +380342723824 \\ ${ }^{2}$ Університет митної справи та фінансів, \\ вул. В.Вернадського, 2/4, корп.1, м. Дніпро, 49000, Україна, е-таil: petrusigor@yahoo.com
}

Методом добутку областей, узагальненому на матричні оператори розсіювання, отримано строгий розв'язок задачі дифракції мод на індуктивному і ємнісному штирях в прямокутному хвилеводі. Квадратна область взаємодії мод, яка містить провідний циліндр, розглядається як загальна частина декількох допоміжних областей, що допускають відокремлення змінних в хвильовому рівнянні. У цій області зв'язку комплексна амплітуда поля представлена у вигляді суперпозиції циліндричних хвиль, породжених штирем, $\mathrm{i}$ хвильових мод, які поширюються від плоских граничних поверхонь. Застосування техніки матричних операторів призводить до математичної моделі у вигляді операторного перетворення (типу "правила складання тангенсів") для узагальненої матриці розсіювання. Аналітично доведена коректність отриманої матричної моделі і безумовна збіжність проекційних наближень до точного рішення. Приведені результати чисельного дослідження коефіцієнта відбиття основної моди хвилеводу для ємнісного штиря в широкому діапазоні зміни частоти і геометрії області зв'язку.

Ключові слова: оператори розсіювання, метод добутку областей, круговий штир у хвилеводі.

Методом произведения областей, обобщенном на матричные операторы рассеяния, получено строгое решение задачи дифракции мод на индуктивном и емкостном штырях в прямоугольном волноводе. Квадратная область взаимодействия мод, содержащая проводящий цилиндр, рассматривается как общая часть нескольких вспомогательных областей, допускающих разделение переменных в волновом уравнении. В этой области связи комплексная амплитуда поля представлена в виде суперпозиции цилиндрических волн, порождаемых штырем, и волноводных мод, распространяющихся от плоских граничных поверхностей. Применение техники матричных операторов приводит к математической модели в виде операторного преобразования (типа “правила сложения тангенсов”) для обобщенной матрицы рассеяния. Аналитически доказана корректность полученной матричной модели и безусловная сходимость проекционных приближений к точному решению. Приведены результаты численного исследования коэффициента отражения основной моды волновода для емкостного штыря в широком диапазоне изменения частоты и геометрии области связи. Ключевые слова: операторы рассеяния, метод произведения областей, круговой штырь в волноводе.

By the method of product fields, generalized to matrix scattering operators, a strict solution to the problem of diffraction modes on inductive and capacitive pins in a rectangular waveguide is obtained. The square interaction region of the mod, which contains the conducting cylinder, is considered as a common part of several auxiliary regions that allow the separation of variables in the wave equation. In this area of communication, the complex amplitude of the field is represented as a superposition of cylindrical waves generated by the pin and wave modes that extend from the plane boundary surfaces. The application of matrix operator technology results in a mathematical model in the form of an operator transform (such as the "rules for drawing up tangents") for a generalized scattering matrix. The correctness of the resulting matrix model and the unconditional convergence of projective approximations to an exact solution are proved analytically. The results of numerical research of the coefficient of reflection of the main waveguide mode for capacitive pin in the wide range of frequency variation and geometry of the. communication domain are presented.

Keywords: scattering operators, region production method, circular pin in waveguide 


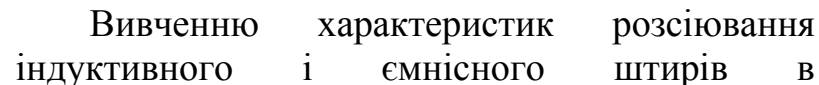
прямокутному хвилеводі присвячено значне число робіт (див, напр. [1-8] і цитовану там літературу), в яких застосовувалися різноманітні методи електродинамічного аналізу. У піонерських дослідженнях (див, напр. [1-3]) було цілком виправдано використання придатних лише для вузького діапазону довжини хвиль наближених методик, точність яких критично залежить від товщини і місця розташування циліндричного розсіювача. Автори подальших робіт, проте, традиційно використали отримані в [2, 3] дані для тестування методів, що розвиваються ними. Мабуть, це пов'язано 3 відсутністю еталонного строгого рішення задачі, а також 3 тим, що не завжди використовувані методи аналізу були цілком строго математично обгрунтовані (див, напр. [4, 6]).

У цій роботі представлений альтернативний розв'язок канонічної задачі дифракції мод на круговому металевому циліндрі в прямокутному хвилеводі, який, зокрема, вільний від згаданих вище обмежень. Ми математично строго обгрунтовуємо коректність отриманої матричної моделі і аналітично доводимо сильну збіжність проекційних наближень до істинного розв'язку.

В процесі побудови розв'язку використовуються базові геометричні ідеї методу добутку областей (МПО) [9-11]. А саме, виділяється область взаємодії хвиль (так звана область зв'язку), що містить задане циліндричне включення, яка розглядається як загальна частина допоміжних областей 3 геометрією, що допускає розділення змінних в рівнянні Гельмгольца. Це дає можливість отримати ефективне представлення для досліджуваної комплексної амплітуди електромагнітного поля.

У цій роботі ми також використовуємо нове формулювання завдання дифракції, що відповідає методу матричних операторів [12], i необхідний у зв'язку з цим новий математичний формалізм. А саме, ми розглядаємо розсіювання на циліндрі повного (тобто нескінченного) набору мод заданої поляризації. Математично це еквівалентно заміні коефіцієнтів Фур'є в рядах по власних функціях на елементи шуканих матричних операторів розсіювання. При цьому традиційні нескінченні системи лінійних алгебраїчних рівнянь зникають, а підсумкова матрична модель набирає вигляду операторного перетворення для узагальненої матриці розсіювання [12-14]. Для досліджуваного завдання розсіювання мод ми отримуємо операторне співвідношення, пов'язане 3 суперпозицією двох перетворень Келі. Використання властивостей цього співвідношення дозволяє довести існування, єдиність i стійкість розв'язку матричного рівняння.

Відмітимо, що для двох можливих поляризацій електромагнітної хвилі джерела ми розглядаємо узагальнену матрицю розсіювання, пов'язану 3 відповідною компонентою електричного поля.

У тексті статті фігурними дужками позначаються нескінченні множини, квадратні дужки застосовуються для формування операторних матриць, а круглі дужки використані для запису скалярного добутку функцій. Окрім цього, ці дужки звичайним способом застосовуються для ранжирування алгебраїчних дій. У кожному випадку сенс використання дужок зрозумілий з контексту і не призводить до колізій.

\section{ПОСТАНОВКА ЗАДАЧІ}

Контур досліджуваної неоднорідності і використовувані системи координат показані на рис. 1. Розглядається прямокутний хвилевод розмірами $a \times 2 b$, у якому паралельно вісі $O y$ розташований провідний круговий циліндр висотою $a$ i радіусом $r<b$. Вісь циліндра віддалена від вертикальної стінки на відстань $d>r$. Хвилевод розділений на три часткові підобласті: два напів нескінченні плеча 1, 3 і квадратну область зв'язку 2, що містить розсіювач. 


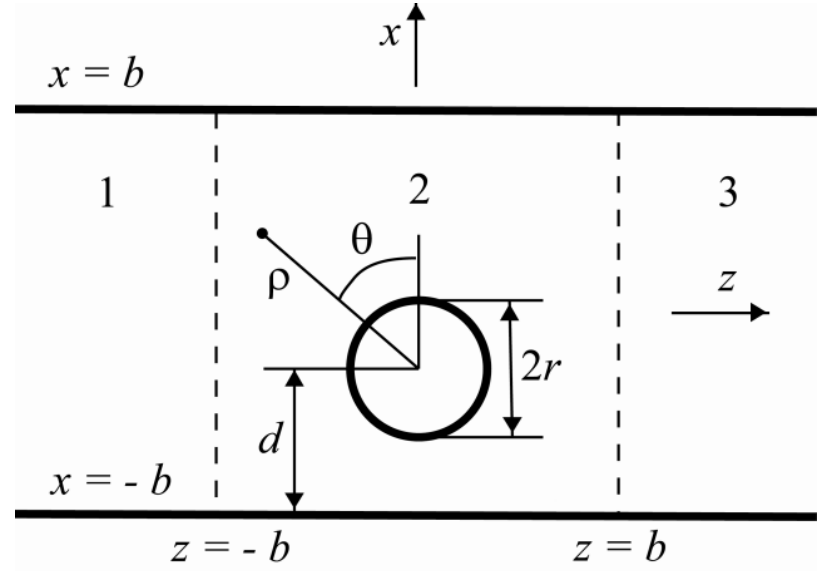

Рисунок 1 - Геометрія задачі

Вважаємо, що в підобластях 1 i 3 розташовані незалежні джерела поля $\alpha$ и $\beta$, відповідно, які генерують монохроматичні поля частотою $\omega$. В результаті на стержень падають ліворуч i праворуч електромагнітні хвилі скінченої потужності, поле яких складає нескінченний набір хвилеводних мод 3 будьяким відомим розподілом амплітуд. Нижче ми паралельно розглядаємо дифракцію $L M_{m 0}, m=1,2, \ldots$, і $L E_{n 1}, n=0,1,2, \ldots$, мод, як найбільш важливих для мікрохвильової техніки, відмічаючи відповідним чином використовувані формули.

Залежно від заданої поляризації хвилі, що падає, запишемо $y$-компоненту поля у вигляді

$$
\begin{gathered}
E_{y}=U(x, z) \exp (i \omega t) \quad(L M) \\
H_{y}=U(x, z) \sin \left(\frac{\pi y}{a}\right) \exp (i \omega t) \quad(L E)
\end{gathered}
$$

а $k=\sqrt{\varepsilon_{0} \mu_{0}} \omega=\frac{2 \pi}{\lambda} \quad$ за сутністю хвильове число.

Згідно методу матричних операторів [12-14] подамо комплексну амплітуду компоненти поля (1) у вигляді скалярного добутку векторів $U=\mathbf{b} \cdot \mathbf{u}(x, z)$, де нескінченний вектор-рядок $\mathbf{b}={ }^{\alpha} \mathbf{b}$ або $\mathbf{b}={ }^{\beta} \mathbf{b}$ характеризує джерело поля i, отже, $є$ будь-яким заданим елементом стандартного Гільбертового простору послідовностей, ${ }^{\alpha, \beta} \mathbf{b} \in \ell_{2}$. Функція $\mathbf{u}(x, z)$, представлена нескінченним вектор-стовпцем, повинна задовольняти однорідним граничним умовам Діріхлє $(L M)$ або Неймана $(L E)$ на провідних поверхнях, умовам неперервності тангенціальних складових поля на межах виділених часткових підобластей, умові на нескінченності для хвилеводів, а також умові скінченності енергії в області визначення поля.

Дифрагуючі хвилеводні моди задані повними ортонормованими системами поперечних власних функцій, об'єднаними у вектор-стовпець

де комплексна амплітуда $U \in$ розв'язком двовимірного рівняння Гельмгольца

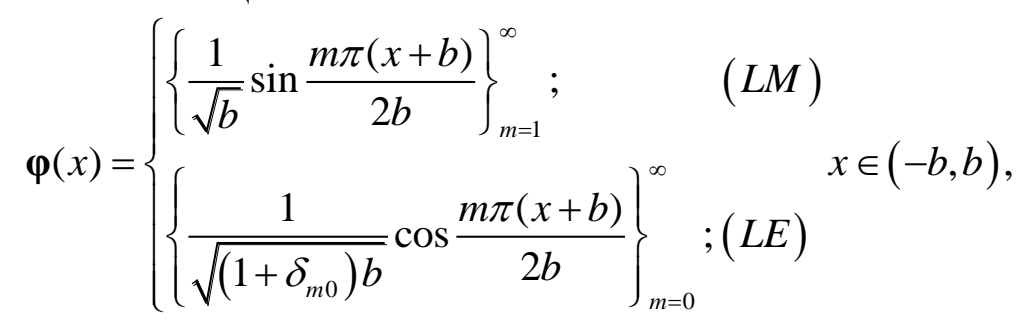

так що тензорно-скалярний добуток цих векторфункцій дорівнює тотожному матричному операторові, $\left(\boldsymbol{\varphi}, \boldsymbol{\varphi}^{T}\right)_{(-b, b)}=\mathbf{I}$. Тут і далі скрізь транспонування. Постійні поширення цих мод $\gamma_{m}=\sqrt{\left(\frac{m \pi}{2 b}\right)^{2}-\chi^{2}}, \quad m=(0), 1,2, \ldots$, 
породжують, у свою чергу, діагональний матричний "оператор подібності"

$\mathbf{I}_{\gamma}=\left\{\delta_{m n} \gamma_{m}\right\}_{m, n=(0) 1}^{\infty}$.

У формулах (3) i (5) $\delta_{m n} \in$ символом Кронекера. У виразі (4) вибрана така гілка квадратного кореня, що $\operatorname{Re} \gamma_{m} \geq 0$ и $\operatorname{Im} \gamma_{m} \geq 0$, причому критичні частоти хвилеводу

$$
\begin{aligned}
& { }^{s} \mathbf{u}^{(1)}=\left\{\begin{array}{l}
{\left[\mathbf{I}_{\gamma}^{-1 / 2} \mathbf{E}(-z-a) \pm{ }^{\alpha} \mathbf{R} \mathbf{I}_{\gamma}^{-1 / 2} \mathbf{E}(z+a)\right] \boldsymbol{\varphi}(x) ; \quad s=\alpha, \quad\left(\begin{array}{l}
L M \\
L E
\end{array}\right)} \\
{ }^{\beta} \mathbf{T} \mathbf{I}_{\gamma}^{-1 / 2} \mathbf{E}(z+a) \boldsymbol{\varphi}(x) ; \quad s=\beta,
\end{array}\right. \\
& { }^{s} \mathbf{u}^{(3)}=\left\{\begin{array}{l}
{ }^{\alpha} \mathbf{T} \mathbf{I}_{\gamma}^{-1 / 2} \mathbf{E}(-z+a) \boldsymbol{\varphi}(x) ; \quad s=\alpha ; \\
{\left[\mathbf{I}_{\gamma}^{-1 / 2} \mathbf{E}(z-a) \pm{ }^{\beta} \mathbf{R} \mathbf{I}_{\gamma}^{-1 / 2} \mathbf{E}(-z+a)\right] \boldsymbol{\varphi}(x) ; \quad s=\beta,\left(\begin{array}{l}
L M \\
L E
\end{array}\right)}
\end{array}\right.
\end{aligned}
$$

де

$$
\mathbf{E}(\zeta)=\left\{\delta_{m n} \exp \left(\gamma_{m} \zeta\right)\right\}_{m, n=(0) 1}^{\infty}
$$

$\epsilon$ діагональним матричним оператором, що описує розповсюдження мод.

Задача полягає у пошуку матричних операторів відображення, що входять у формули (6) i (7) $\quad{ }^{\alpha, \beta} \mathbf{R}: \ell_{2} \rightarrow \ell_{2} \quad$ і проходження ${ }^{\alpha, \beta} \mathbf{T}: \ell_{2} \rightarrow \ell_{2}$, або, що те саме, узагальненої матриці розсіювання чотириполюсника

$\hat{\mathbf{S}}=\left[\begin{array}{cc} \pm^{\alpha} \mathbf{R} & { }^{\alpha} \mathbf{T} \\ { }^{\beta} \mathbf{T} & \pm^{\beta} \mathbf{R}\end{array}\right] \quad\left(\begin{array}{l}L M \\ L E\end{array}\right)$

тобто найпростішої $2 \times 2$ операторної матриці $\ell_{2}^{2} \rightarrow \ell_{2}^{2}$.

Слідуючи МПО $[10,11]$, представимо функцію

$$
\mathbf{u}^{(2)}(x, z)=\mathbf{u}_{r}(\rho, \theta)+\sum_{m=1}^{4} \mathbf{u}_{m}(x, z)
$$

де вектор-функції $\mathbf{u}_{r}$ и $\mathbf{u}_{m}, m=\overline{1,4}$, визначені у допоміжних областях, перетин яких утворює область зв'язку 2, а саме:

$\{(\theta, \rho): \rho>r,-\pi<\theta \leq \pi\}$, $\{(x, z): x>-b,|z|<b\},\{(x, z):|x|<b, z<b\}$, $\{(x, z): x<b,|z|<b\}$ и $\{(x, z):|x|<b, z>-b\}$.

Відокремивши змінні в рівнянні Гельмгольца у вказаних системах координат, отримаємо у матрично-векторній формі наступні розклади

$\mathbf{u}^{(2)}$ сумою

$$
\begin{gathered}
\mathbf{u}_{r}=\mathbf{F} \mathbf{I}_{\gamma}^{-1 / 2} \mathbf{H}(\rho) \mathbf{c}(\theta)+\mathbf{G} \mathbf{I}_{\gamma}^{-1 / 2} \mathbf{H}(\rho) \mathbf{s}(\theta) ; \\
\mathbf{u}_{1}=\mathbf{D}_{1} \mathbf{I}_{\gamma}^{-1 / 2} \mathbf{E}(-x-a) \boldsymbol{\psi}(z) ; \quad \mathbf{u}_{3}=\mathbf{D}_{3} \mathbf{I}_{\gamma}^{-1 / 2} \mathbf{E}(x-a) \boldsymbol{\psi}(z) ; \\
\mathbf{u}_{2}=\mathbf{D}_{2} \mathbf{I}_{\gamma}^{-1 / 2} \mathbf{E}(z-a) \boldsymbol{\varphi}(x) ; \quad \mathbf{u}_{4}=\mathbf{D}_{4} \mathbf{I}_{\gamma}^{-1 / 2} \mathbf{E}(-z-a) \boldsymbol{\varphi}(x),
\end{gathered}
$$

по повних системах ортонормованих функцій,

зібраних у вектор-стовпці

$$
\mathbf{c}(\theta)=\left\{\frac{1}{\sqrt{\left(1+\delta_{m 0}\right) \pi}} \cos (m \theta)\right\}_{m=0}^{\infty} ; \quad \mathbf{s}(\theta)=\left\{\frac{1}{\sqrt{\pi}} \sin (m \theta)\right\}_{m=1}^{\infty}, \quad \theta \in(-\pi, \pi],
$$




$$
\boldsymbol{\psi}(z)=\left\{\frac{1}{\sqrt{\left(1+\delta_{m 0}\right) b}} \cos \frac{m \pi(z+b)}{2 b}\right\}_{m=0}^{\infty}, \quad z \in(-b, b),
$$

і таких, що $\left(\mathbf{c}, \mathbf{c}^{T}\right)_{(-\pi, \pi]}=\mathbf{I},\left(\mathbf{s}, \mathbf{s}^{T}\right)_{(-\pi, \pi]}=\mathbf{I}$, $\left(\mathbf{c}, \mathbf{s}^{T}\right)_{(-\pi, \pi]}=\mathbf{0}$ и $\left(\boldsymbol{\psi}, \boldsymbol{\psi}^{T}\right)_{(-b, b)}=\mathbf{I}$.

У формулі (11) введений діагональний матричний оператор

$$
\mathbf{H}(\rho)=\left\{\delta_{m n} \frac{H_{m}^{(2)}(\chi \rho)}{H_{m}^{(2)}(\chi r)}\right\}_{m, n=0(1)}^{\infty},
$$

що описує поширення циліндричних хвиль. Тут $H_{m}^{(2)}(\zeta)$ - функція Ханкеля другого роду. У представленнях (11)-(13) оператори $\mathbf{F , G ~ и ~}$ $\mathbf{D}_{m}, m=\overline{1,4}$, залежать від індексу джерела поля $\alpha$ або $\beta$ (який ми опускаємо 3 метою спрощення запису формул) і надалі підлягають визначенню.

Відмітимо, що вектор-функції $\mathbf{u}_{m}, m=\overline{1,4}$, вибрані так, щоб задовольнити наступним граничним умовам в області зв'язку 2 :

$$
\begin{gathered}
\frac{\partial \mathbf{u}_{1}}{\partial z}=\frac{\partial \mathbf{u}_{3}}{\partial z}=0, \quad z= \pm b, \\
\left\{\begin{array}{l}
\mathbf{u}_{2}=\mathbf{u}_{4}=0 ; \quad(L M) \\
\frac{\partial \mathbf{u}_{2}}{\partial x}=\frac{\partial \mathbf{u}_{4}}{\partial x}=0, \quad(L E)
\end{array} \quad x= \pm b .\right.
\end{gathered}
$$

Використовуване представлення (10) величини $\mathbf{u}^{(2)}$ очевидно можливе. 3 одного боку, побудована таким чином вектор-функція $\mathbf{u}^{(2)}$ тотожно задовольняє рівнянню Гельмгольца в області 2. 3 іншої - описана конструкція породжує на кожній частині межі цієї області повну систему функцій, що дозволяє задовільнити будь-яким допустимим граничним умовам.

Випишемо тепер в явному вигляді умови, що накладаються на межі двозв'язної області взаємодії хвиль 2. На тих їх частинах, які відповідають провідним поверхням, 3 урахуванням умов (17) і (18) ми отримуємо

$$
\begin{cases}\mathbf{u}_{r}+\mathbf{u}_{1}+\mathbf{u}_{3}=0 ; & x= \pm b \\ \mathbf{u}_{r}+\sum_{m=1}^{4} \mathbf{u}_{m}=0 ; & \rho=r\end{cases}
$$

$$
\begin{cases}\frac{\partial}{\partial x}\left(\mathbf{u}_{r}+\mathbf{u}_{1}+\mathbf{u}_{3}\right)=0 ; & x= \pm b ; \\ \frac{\partial}{\partial \rho}\left(\mathbf{u}_{r}+\sum_{m=1}^{4} \mathbf{u}_{m}\right)=0 ; & \rho=r .\end{cases}
$$

На спільних межах підобластей $z=\mp b$ умови неперервності тангенціальних компонент електромагнітного поля призводять до рівності

$$
\begin{aligned}
\mathbf{u}^{(q)} & =\mathbf{u}_{r}+\sum_{m=1}^{4} \mathbf{u}_{m} ; \\
\frac{\partial \mathbf{u}^{(q)}}{\partial z} & =\frac{\partial}{\partial z}\left(\mathbf{u}_{r}+\mathbf{u}_{2}+\mathbf{u}_{4}\right) ;
\end{aligned} \quad q= \begin{cases}1, & z=-b ; \\
3, & z=+b\end{cases}
$$

незалежно від поляризації падаючої хвилі.

\section{ПОБУДОВА ОПЕРАТОРНОЇ МОДЕЛІ}

У подальшому аналізі ключову роль грають операторні матриці

$$
\hat{\mathbf{S}}_{0}=\left[\begin{array}{cc}
\mathbf{0} & \mathbf{E}(-2 b) \\
\mathbf{E}(-2 b) & \mathbf{0}
\end{array}\right], \quad \hat{\mathbf{J}}=\left[\begin{array}{cc}
\mathbf{I} & \mathbf{0} \\
\mathbf{0} & -\mathbf{I}
\end{array}\right],
$$

перша 3 яких $\epsilon$ не що інше, як узагальнена матриця розсіювання порожнистого відрізку хвилеводу завдовжки $2 b$, а друга є канонічною симетрією простору $\ell_{2}^{2}$. Введемо також "амплітуду розсіювання" вказаного відрізку хвилеводу за формулами

$$
\hat{\mathbf{A}}=\hat{\mathbf{I}}+\hat{\mathbf{S}}_{0} \Leftrightarrow \hat{\mathbf{J}} \hat{\mathbf{A}} \hat{\mathbf{J}}=\hat{\mathbf{I}}-\hat{\mathbf{S}}_{0},
$$

де $\hat{\mathbf{I}}$ - одиничний оператор у просторі $\ell_{2}^{2}$. Обернені до матриць (23) оператори будуть обмеженими за умови $\pm 1 \notin \sigma\left(\hat{\mathbf{S}}_{0}\right)$, яке обумовлюється в наступному розділі. Крім того, нам знадобляться діагональні операторні матриці

$$
\hat{\mathbf{I}}_{\gamma}=\left[\begin{array}{cc}
\mathbf{I}_{\gamma} & \mathbf{0} \\
\mathbf{0} & \mathbf{I}_{\gamma}
\end{array}\right], \quad \hat{\mathbf{H}}=\left[\begin{array}{cc}
\mathbf{H} & \mathbf{0} \\
\mathbf{0} & \mathbf{H}
\end{array}\right], \quad \hat{\boldsymbol{\Xi}}= \begin{cases}\hat{\mathbf{I}} ; & (L M) \\
\hat{\mathbf{H}}^{-1}, & (L E)\end{cases}
$$

перша 3 яких $\epsilon$, вочевидь, (двостороннє) оборотною за умови виключення 3 розгляду критичних частот хвилеводу.

Вважаючи, що джерело $\beta$ вимкнено, ми знайдемо першу половину шуканої матричної 
моделі. 3 цією метою підставимо розклад (11) (13) в граничні умови на стінках хвилеводу $x= \pm b$, формула (19) або (20), і скористаємося властивістю ортогональності функції $\boldsymbol{\psi}(z)$. Це

дає наступні співвідношення для знову сформованих $1 \times 2$ операторних матриць

$$
\begin{array}{r}
{[\mathbf{F}, \mathbf{G}] \hat{\mathbf{B}}+\left[\mathbf{D}_{1}, \mathbf{D}_{3}\right] \hat{\mathbf{A}}=0,(L M) \text { або }} \\
{[\mathbf{F}, \mathbf{G}] \hat{\mathbf{B}}-\left[\mathbf{D}_{1}, \mathbf{D}_{3}\right] \hat{\mathbf{J}} \hat{\mathbf{A}}=0 .(L E)}
\end{array}
$$

Тут позначено

$$
\begin{aligned}
\hat{\mathbf{B}}=\hat{\mathbf{I}}_{\gamma}^{-1 / 2} & {\left[\begin{array}{ll}
\mathbf{B}_{c}^{(-)} & \mathbf{B}_{c}^{(+)} \\
\mathbf{B}_{s}^{(-)} & \mathbf{B}_{s}^{(+)}
\end{array}\right] \hat{\mathbf{I}}_{\gamma}^{ \pm 1 / 2}, \quad\left(\begin{array}{l}
L M \\
L E
\end{array}\right) ; \quad \mathbf{B}_{c, s}^{( \pm)}=\left(\mathbf{f}_{c, s}, \boldsymbol{\psi}^{T}\right)_{(-b, b)} ; } \\
\mathbf{f}_{c, s}= & \left\{\begin{array}{l}
\left.\mathbf{g}_{c, s}\right|_{x= \pm b} ; \quad(L M) \\
\left.\frac{\partial \mathbf{g}_{c, s}}{\partial x}\right|_{x= \pm b}, \quad(L E)
\end{array} \quad \mathbf{g}_{c}=\mathbf{H}(\rho) \mathbf{c}(\theta) ; \quad \mathbf{g}_{s}=\mathbf{H}(\rho) \mathbf{s}(\theta) .\right.
\end{aligned}
$$

Далі, підставимо розклад (11) - (13) в граничну умову на циліндрі $\rho=r$, формула (19) або (20), і скористаємося ортогональністю функцій $\mathbf{c}(\theta)$ i $\mathbf{s}(\theta)$. Це дає рівність

$$
\begin{aligned}
& \hat{\mathbf{C}}_{\eta}=\hat{\mathbf{I}}_{\gamma}^{-1 / 2}\left[\begin{array}{cc}
{ }^{\eta} \mathbf{C}_{c}^{(-)} & { }^{\eta} \mathbf{C}_{s}^{(-)} \\
{ }^{\eta} \mathbf{C}_{c}^{(+)} & { }^{\eta} \mathbf{C}_{s}^{(+)}
\end{array}\right] \hat{\Xi} \hat{\mathbf{I}}_{\gamma}^{1 / 2}, \quad \eta=\psi, \varphi ; \\
& { }^{\eta} \mathbf{C}_{c}^{( \pm)}=\left(\mathbf{f}_{\eta}^{( \pm)}, \mathbf{c}^{T}\right)_{(-\pi, \pi]},{ }^{\eta} \mathbf{C}_{s}^{( \pm)}=\left(\mathbf{f}_{\eta}^{( \pm)}, \mathbf{s}^{T}\right)_{(-\pi, \pi]} ;
\end{aligned}
$$

$$
[\mathbf{F}, \mathbf{G}]+\left[\mathbf{D}_{1}, \mathbf{D}_{3}\right] \hat{\mathbf{C}}_{\psi}+\left[\mathbf{D}_{4}, \mathbf{D}_{2}\right] \hat{\mathbf{C}}_{\varphi}=0,
$$

в якому введені позначення

$$
\mathbf{f}_{\eta}^{( \pm)}=\left\{\begin{array}{ll}
\left.\mathbf{g}_{\eta}^{( \pm)}\right|_{\rho=r} ; & (L M) \\
\left.\frac{\partial \mathbf{g}_{\eta}^{( \pm)}}{\partial \rho}\right|_{\rho=r}, & (L E)
\end{array} \quad \mathbf{g}_{\eta}^{( \pm)}=\left\{\begin{array}{l}
\mathbf{E}( \pm x-b) \boldsymbol{\psi}(z), \eta=\psi \\
\mathbf{E}( \pm z-b) \boldsymbol{\varphi}(x), \eta=\varphi
\end{array}\right.\right.
$$

Залишилося застосувати умови зшивання полів (21), в які потрібно підставити розклад (6), (7) і ортогональності функції $\varphi(x)$. В підсумку знаходимо

(11) - (13), після чого скористатися властивістю

$$
\begin{aligned}
& -\left[ \pm{ }^{\alpha} \mathbf{R},{ }^{\alpha} \mathbf{T}\right]+[\mathbf{F}, \mathbf{G}] \hat{\mathbf{K}}+\left[\mathbf{D}_{1}, \mathbf{D}_{3}\right] \hat{\mathbf{L}}+\left[\mathbf{D}_{4}, \mathbf{D}_{2}\right] \hat{\mathbf{A}}=[\mathbf{I}, \mathbf{0}], \quad\left(\begin{array}{l}
L M \\
L E
\end{array}\right) \\
& -\left[ \pm{ }^{\alpha} \mathbf{R},{ }^{\alpha} \mathbf{T}\right] \hat{\mathbf{J}}+[\mathbf{F}, \mathbf{G}] \hat{\mathbf{W}}-\left[\mathbf{D}_{4}, \mathbf{D}_{2}\right] \hat{\mathbf{J}} \hat{\mathbf{A}}=-[\mathbf{I}, \mathbf{0}]\left(\begin{array}{l}
L M \\
L E
\end{array}\right)
\end{aligned}
$$

Тут позначено

$$
\begin{gathered}
\hat{\mathbf{K}}=\hat{\mathbf{I}}_{\gamma}^{-1 / 2}\left[\begin{array}{ll}
\mathbf{K}_{c}^{(-)} & \mathbf{K}_{c}^{(+)} \\
\mathbf{K}_{s}^{(-)} & \mathbf{K}_{s}^{(+)}
\end{array}\right] \hat{\mathbf{I}}_{\gamma}^{1 / 2}, \hat{\mathbf{L}}=\hat{\mathbf{I}}_{\gamma}^{-1 / 2}\left[\begin{array}{cc}
\mathbf{L}_{1}^{(-)} & \mathbf{L}_{3}^{(-)} \\
\mathbf{L}_{1}^{(+)} & \mathbf{L}_{3}^{(+)}
\end{array}\right] \hat{\mathbf{I}}_{\gamma}^{1 / 2}, \hat{\mathbf{W}}=\hat{\mathbf{I}}_{\gamma}^{-1 / 2}\left[\begin{array}{cc}
\mathbf{W}_{c}^{(-)} & \mathbf{W}_{c}^{(+)} \\
\mathbf{W}_{s}^{(-)} & \mathbf{W}_{s}^{(+)}
\end{array}\right] \hat{\mathbf{I}}_{\gamma}^{-1 / 2} \\
\mathbf{K}_{c, s}^{( \pm)}=\left(\left.\mathbf{g}_{c, s}\right|_{z= \pm b}, \boldsymbol{\varphi}^{T}\right)_{(-b, b)} ; \mathbf{W}_{c, s}^{( \pm)}=\left(\left.\frac{\partial \mathbf{g}_{c, s}}{\partial z}\right|_{z= \pm b}, \boldsymbol{\varphi}^{T}\right)_{(-b, b)} ; \\
\mathbf{L}_{j}^{( \pm)}=\left(\left.\mathbf{g}_{\psi}\right|_{z= \pm b}, \boldsymbol{\varphi}^{T}\right)_{(-b, b)}, j= \begin{cases}1, & z=-b ; \\
3, & z=b .\end{cases}
\end{gathered}
$$

Співвідношення (25), (27), (29) і (30) утворюють систему з чотирьох рівнянь відносно чотирьох невідомих $1 \times 2$ операторних матриць.
Виключаючи з цієї системи усі невідомі, окрім матриці, яка містить шукані оператори розсіювання, формально знаходимо 


$$
-\left[ \pm^{\alpha} \mathbf{R},{ }^{\alpha} \mathbf{T}\right]\left(\hat{\mathbf{I}}-\hat{\mathbf{S}}_{0} \hat{\mathbf{V}}\right)=\frac{1}{2}[\mathbf{I}, \mathbf{0}] \hat{\mathbf{A}}^{-1}(\hat{\mathbf{I}}-\hat{\mathbf{J}}) \hat{\mathbf{F}}_{0}+\frac{1}{2}[\mathbf{I}, \mathbf{0}] \hat{\mathbf{A}}^{-1}(\hat{\mathbf{I}}+\hat{\mathbf{J}}) \hat{\mathbf{\Lambda}} \hat{\mathbf{F}}_{0}, \quad\left(\begin{array}{l}
L M \\
L E
\end{array}\right)
$$

де

$$
\begin{aligned}
& \hat{\mathbf{V}}=\hat{\mathbf{F}}_{0}^{-1} \hat{\boldsymbol{\Lambda}} \hat{\mathbf{F}}_{0} ; \quad \hat{\mathbf{\Lambda}}=\hat{\mathbf{C}}_{\varphi} \hat{\mathbf{N}}^{-1} \hat{\mathbf{M}}^{(+)} ; \quad \hat{\mathbf{F}}_{0}=\hat{\mathbf{I}}-\hat{\mathbf{S}}_{0}^{2} ; \\
& \hat{\mathbf{N}}=\hat{\mathbf{M}}^{(-)} \hat{\mathbf{C}}_{\varphi}+2\left(\hat{\mathbf{I}}+\hat{\mathbf{B}} \hat{\mathbf{A}}^{-1} \hat{\boldsymbol{\Theta}} \hat{\mathbf{C}}_{\psi}\right) ; \quad \hat{\boldsymbol{\Theta}}=\left\{\begin{array}{rr}
-\hat{\mathbf{I}}, & (L M) \\
\hat{\mathbf{J}} ; & (L E)
\end{array}\right. \\
& \hat{\mathbf{M}}^{( \pm)}=\hat{\mathbf{W}} \hat{\mathbf{A}}^{-1} \hat{\mathbf{J}} \pm\left(\hat{\mathbf{K}}+\hat{\mathbf{B}} \hat{\mathbf{A}}^{-1} \hat{\boldsymbol{\Theta}} \hat{\mathbf{L}}\right) \hat{\mathbf{A}}^{-1} .
\end{aligned}
$$

Тепер ті ж міркування необхідно повторити при включеному джерелі поля $\beta$, але вимкненому джерелі $\alpha$. Очевидно, співвідношення (25) і (27), які виходять 3

$$
-\left[{ }^{\beta} \mathbf{T}, \pm^{\beta} \mathbf{R}\right]\left(\hat{\mathbf{I}}-\hat{\mathbf{S}}_{0} \hat{\mathbf{V}}\right)=\frac{1}{2}[\mathbf{0}, \mathbf{I}] \hat{\mathbf{A}}^{-1}(\hat{\mathbf{I}}+\hat{\mathbf{J}}) \hat{\mathbf{F}}_{0}+\frac{1}{2}[\mathbf{0}, \mathbf{I}] \hat{\mathbf{A}}^{-1}(\hat{\mathbf{I}}-\hat{\mathbf{J}}) \hat{\mathbf{\Lambda}} \hat{\mathbf{F}}_{0} . \quad\left(\begin{array}{l}
L M \\
L E
\end{array}\right)
$$

3 урахуванням визначення (9) матричне об'єднання рівності (32) і (34) дає нам компактний вид шуканої операторної моделі

$$
\hat{\mathbf{S}}\left(\hat{\mathbf{I}}-\hat{\mathbf{S}}_{0} \hat{\mathbf{V}}\right)=\hat{\mathbf{S}}_{0}-\hat{\mathbf{V}}
$$

до дослідження властивостей якої ми і приступаємо.

\section{КОРЕКТНІСТЬ МАТРИЧНОЇ МОДЕЛІ}

Доведемо існування, єдиність і стійкість рішення знайденого операторного рівняння (35), яке услід за м. Вейлем [15] іменуватимемо співвідношенням типу "правила складання тангенсів". При цьому ми використовуємо відомі властивості узагальнених матриць розсіювання $\hat{\mathbf{S}}, \quad \hat{\mathbf{S}}_{0}$ i $і$ іх спектрів, а також узагальнених матриць опору $\hat{\mathbf{Z}}, \quad \hat{\mathbf{Z}}_{0} \quad \mathrm{i}$ провідності $\hat{\mathbf{Y}}, \hat{\mathbf{Y}}_{0}$ [16]. Характерна дуальність властивостей операторів, що входить у формулу (35), полягає в наступному: якщо для заданого кожним своїм елементом матричного оператора $\hat{\mathbf{V}}$ невідома локалізація спектру, яка нас цікавить $\sigma(\hat{\mathbf{V}})$, те основні характеристики повного спектру шуканого оператора розсіювання $\sigma(\hat{\mathbf{S}})$ повністю визначені законом збереження енергії в узагальненій формі [12, 17]. Взаємозв'язок цих двох операторів у вигляді перетворення (35) і відомі характеристики граничних умов, залишаться по виду попередніми. Враховуючи зміни в поданнях (6), (7) і проводячи аналогічний виклад, формально знаходимо

спектру $\sigma\left(\hat{\mathbf{S}}_{0}\right)$ дозволяють знайти усі їх необхідні властивості.

Кінцевий об'єм області зв'язку 2 утворює (відкритий) резонатор; отже, необхідно виключити 3 розгляду усі особливі точки частотного діапазону. Окрім обумовленої вище умови виключення критичних частот хвилеводу, ми видаляємо з частотної вісі наступну зліченну множину точок:

які задаються рівністю $\chi_{m n}=\pi \sqrt{m^{2}+n^{2}} / 2 b$, $m, n=(0) 1,2, \ldots$, частоти резонансу у вільному від розсіювача області зв'язку, для яких $\pm 1 \in \sigma\left(\hat{\mathbf{S}}_{0}\right)$ (відмітимо також, що для цих частот оператор $\hat{\mathbf{L}}$ не визначений);

частоти резонансу в області зв'язки у присутності циліндричного стержня, для яких $\pm 1 \in \sigma(\hat{\mathbf{S}})$.

Для цих останніх резонансних частот із закону збереження потужності яка коливається, виходить $\quad \hat{\mathbf{S}}^{2}=\hat{\mathbf{I}}$. Тоді 3 формули (35) отримуємо, що спектру $\sigma(\hat{\mathbf{S}})= \pm 1$ відповідає $\sigma(\hat{\mathbf{V}})=\mp 1$ за умови $\pm 1 \notin \sigma\left(\hat{\mathbf{S}}_{0}\right)$.

Знайдемо взаємозв'язок цих спектрів для нерезонансних частот. Для цього перепишемо співвідношення (35) у вигляді 


$$
(\hat{\mathbf{I}} \pm \hat{\mathbf{S}})\left(\hat{\mathbf{I}}-\hat{\mathbf{S}}_{0} \hat{\mathbf{V}}\right)=\left(\hat{\mathbf{I}} \pm \hat{\mathbf{S}}_{0}\right)(\hat{\mathbf{I}} \mp \hat{\mathbf{V}}) .
$$

Нехай спочатку $1 \notin \sigma(\hat{\mathbf{S}})$, тобто $\|\hat{\mathbf{Z}}\|<\infty$. Тоді, виключаючи з (36) оператор $\left(\hat{\mathbf{I}}-\hat{\mathbf{S}}_{0} \hat{\mathbf{V}}\right)$, отримаємо рівність

$$
\hat{\mathbf{Y}}_{V}=\left(\hat{\mathbf{I}}+\hat{\mathbf{Z}}_{0}\right) \hat{\mathbf{Y}}_{0} \hat{\mathbf{Z}}\left(\hat{\mathbf{I}}+\hat{\mathbf{Z}}_{0}\right)^{-1}
$$

де $\hat{\mathbf{Y}}_{V} \epsilon$ перетворення Келі оператора $\hat{\mathbf{V}}$, яке задане рівнянням

$$
\left(\hat{\mathbf{I}}+\hat{\mathbf{Y}}_{V}\right)(\hat{\mathbf{I}}+\hat{\mathbf{V}})=(\hat{\mathbf{I}}+\hat{\mathbf{V}})\left(\hat{\mathbf{I}}+\hat{\mathbf{Y}}_{V}\right)=2 \hat{\mathbf{I}}
$$

$$
\left(\hat{\mathbf{I}}-\hat{\mathbf{S}}_{0} \hat{\mathbf{V}}\right)^{-1}=\frac{1}{2}\left\{\begin{array}{l}
\left(\hat{\mathbf{I}}+\hat{\mathbf{Y}}_{0}\right)\left(\hat{\mathbf{Z}}_{0}+\hat{\mathbf{Z}}\right)(\hat{\mathbf{I}}+\hat{\mathbf{Z}})^{-1},+1 \notin \sigma(\hat{\mathbf{S}}) ; \\
\left(\hat{\mathbf{I}}+\hat{\mathbf{Z}}_{0}\right)\left(\hat{\mathbf{Y}}_{0}+\hat{\mathbf{Y}}\right)(\hat{\mathbf{I}}+\hat{\mathbf{Y}})^{-1},-1 \notin \sigma(\hat{\mathbf{S}}),
\end{array}\right.
$$

і констатувати його обмеженість як наслідок виконання закону збереження енергії..

Таким чином, єдиний розв'язок даної задачі дифракції хвилеводних мод подається формулою

$$
\hat{\mathbf{S}}=\left(\hat{\mathbf{S}}_{0}-\hat{\mathbf{V}}\right)\left(\hat{\mathbf{I}}-\hat{\mathbf{S}}_{0} \hat{\mathbf{V}}\right)^{-1},
$$

справедливою для усіх значень хвилевого числа за винятком дискретного ряду вказаних вище особливих частот.

Відмітимо, що оператор $\hat{\mathbf{S}}_{0} \epsilon$ нерозтягуючим ядерним оператором, тому

$$
\mathbf{P}_{K}=\left\{P_{m n}^{(K)}=\sum_{q=0}^{K-1} \delta_{m q} \delta_{q n}\right\}_{m, n=0}^{\infty}, K=M, N ;
$$

Тут $M \in$ число хвилеводних мод, а $N$ - число циліндричних хвиль, що враховуються при знаходженні проекційних наближень. Таким чином, ми використовуватимемо однаково обрізані системи власних функцій $\mathbf{P}_{M} \boldsymbol{\varphi}(x)$ и $\mathbf{P}_{M} \boldsymbol{\psi}(z)$, що $є$ наслідком вибраної геометрії області зв'язку 2 (інакше кажучи, при виведенні рівняння (35) ми не відрізняли узагальнені матриці розсіяння $\hat{\mathbf{S}}_{0}$ горизонтального відрізку хвилеводу і вертикального відрізку (віртуального) хвилеводу, що мають однакову довжину $2 b)$. Згідно $з$ методом редукції, після знаходження скінченномірного наближення
3 представлення (37) виходить, що $\left\|\hat{\mathbf{Y}}_{V}\right\|<\infty$; тоді із співвідношення (38) витікає обмеженість оберненого оператора

$$
(\hat{\mathbf{I}}+\hat{\mathbf{V}})^{-1}=\frac{1}{2}\left(\hat{\mathbf{I}}+\hat{\mathbf{Y}}_{V}\right),
$$

т.е. $-1 \notin \sigma(\hat{\mathbf{V}})$. Аналогічно знаходимо, що

$1 \notin \sigma(\hat{\mathbf{V}})$, як що $-1 \notin \sigma(\hat{\mathbf{S}})$, тобто $\|\hat{\mathbf{Y}}\|<\infty$.

Для доведення коректності побудованої операторної моделі тепер досить з рівності (36) отримати наступні представлення для оберненого оператора

знайдену модель (35), (41) можна також інтерпретувати як узагальнене рівняння другого роду, для якого справедлива альтернатива Фредгольма.

\section{БЕЗУМОВНА ЗБІЖНІСТЬ НАБЛИЖЕНЬ}

$$
\text { Для знаходження скінченномірних }
$$
матричних наближень $\tilde{\mathbf{S}}$ застосуємо метод редукції рішення рівняння (35). 3 цією метою введемо ортопроектори

$\mathbf{P}_{N-1}=\left\{P_{m n}^{(N-1)}=\sum_{q=1}^{N-1} \delta_{m q} \delta_{q n}\right\}_{m, n=1}^{\infty}$

необхідно виконати граничний перехід $M, N \rightarrow \infty$.

Утворюємо (проекційні) операторні матриці по формулах

$$
\hat{\mathbf{P}}_{M}=\left[\begin{array}{cc}
\mathbf{P}_{M} & \mathbf{0} \\
\mathbf{0} & \mathbf{P}_{M}
\end{array}\right], \quad \hat{\mathbf{P}}_{N}=\left[\begin{array}{cc}
\mathbf{P}_{N} & \mathbf{0} \\
\mathbf{0} & \mathbf{P}_{N-1}
\end{array}\right],
$$

i $\hat{\mathbf{Q}}_{K}=\hat{\mathbf{I}}-\hat{\mathbf{P}}_{K}, K=M, N$. Відмітимо, що в силу особливої структури оператора $\hat{\mathbf{S}}_{0}$ має місце наступне представлення

$$
\widetilde{\mathbf{S}}_{0}=\hat{\mathbf{P}}_{M} \hat{\mathbf{S}}_{0}=\hat{\mathbf{S}}_{0} \hat{\mathbf{P}}_{M}
$$

(так зване співвідношення комутації). Це ж правило справедливо і для операторів

$$
\hat{\mathbf{A}}^{-1}, \hat{\mathbf{J}} \text { и } \hat{\mathbf{A}}^{-1} \hat{\mathbf{J}} \text {. }
$$


Побудова скінченномірної матричної моделі нічим не відрізняється від викладеного вище доведення точного співвідношення (35) i призводить до формули

$$
\tilde{\mathbf{S}}\left(\hat{\mathbf{P}}_{M}-\tilde{\mathbf{S}}_{0} \tilde{\mathbf{V}}\right)=\tilde{\mathbf{S}}_{0}-\tilde{\mathbf{V}},
$$

де позначено

$$
\tilde{\mathbf{V}}=\tilde{\mathbf{F}}_{0}^{-1} \tilde{\mathbf{\Lambda}} \tilde{\mathbf{F}}_{0} ; \quad \tilde{\mathbf{F}}_{0}=\hat{\mathbf{P}}_{M} \hat{\mathbf{F}}_{0}=\hat{\mathbf{P}}_{M}-\tilde{\mathbf{S}}_{0}^{2} .
$$

Аналогічно (46), вирази для $\tilde{\boldsymbol{\Lambda}}$ і інших використовуваних операторів мають вид, визначуваний формулами (33), записаними для кінцевих матриць 3 урахуванням наступних правил усікання :

$$
\begin{aligned}
& \tilde{\mathbf{B}}=\hat{\mathbf{P}}_{N} \hat{\mathbf{B}} \hat{\mathbf{P}}_{M} ; \quad \tilde{\mathbf{W}}=\hat{\mathbf{P}}_{N} \hat{\mathbf{W}} \hat{\mathbf{P}}_{M} ; \quad \tilde{\mathbf{K}}=\hat{\mathbf{P}_{N}} \hat{\mathbf{K}} \hat{\mathbf{P}}_{M} ; \\
& \tilde{\mathbf{C}}_{\varphi}=\hat{\mathbf{P}}_{M} \hat{\mathbf{C}}_{\varphi} \hat{\mathbf{P}}_{N} ; \tilde{\mathbf{C}}_{\psi}=\hat{\mathbf{P}}_{M} \hat{\mathbf{C}}_{\psi} \hat{\mathbf{P}}_{N} ; \tilde{\mathbf{L}}=\hat{\mathbf{P}}_{M} \hat{\mathbf{L}} \hat{\mathbf{P}}_{M} .
\end{aligned}
$$

Далі, повторюючи думку попереднього розділу, але тепер для матричного рівняння (45), знаходимо, що наслідком виконання закону збереження енергії для скінченномірних наближень буде невиродженість $2 M \times 2 M$ матриці $\left(\hat{\mathbf{P}}_{M}-\tilde{\mathbf{S}}_{0} \tilde{\mathbf{V}}\right)$ для будь-яких чисел $M$, $N$ i усіх неособливих точок частотного діапазону. При цій же умові як обернена матриця $\left(\hat{\mathbf{P}}_{M}-\tilde{\mathbf{S}}_{0} \tilde{\mathbf{V}}\right)^{-1}$, так і усі інші матричні оператори (46), (47) будуть обмеженими незалежно від $M$ і $N$ в силу принципу рівномірної обмеженості Банаха - Штейнхауса (див, напр. [18]).

Надалі нас цікавитиме лише проекційна збіжність (або $P$-збіжність) матричних наближень, яка $\epsilon$ оптимально інформативною 3 точки зору додатків (з приводу визначення $P$ збіжності див., напр., [18]).

Використовуючи наближеного розв'язку у формі

$$
\tilde{\mathbf{S}}=\left(\hat{\mathbf{P}}_{M}-\tilde{\mathbf{V}}^{T} \tilde{\mathbf{S}}_{0}\right)^{-1}\left(\tilde{\mathbf{S}}_{0}-\tilde{\mathbf{V}}^{T}\right),
$$

Отримаємо оцінку

$$
\left\|\left(\hat{\mathbf{P}}_{M} \hat{\mathbf{S}}-\tilde{\mathbf{S}}\right) \mathbf{b}^{T}\right\| \leq \text { const } \cdot\left\|\left(\tilde{\boldsymbol{\Lambda}}-\hat{\mathbf{P}}_{M} \hat{\mathbf{\Lambda}}\right) \mathbf{d}^{T}\right\| ; \mathbf{d}^{T}=\hat{\mathbf{F}}_{0}\left(\hat{\mathbf{I}}-\hat{\mathbf{S}}_{0} \hat{\mathbf{V}}\right)^{-1} \mathbf{b}^{T}, \quad \forall \mathbf{b} \in \ell_{2} .
$$

Тобто, доказ сильної $P$ - збіжності шуканих наближень (45) зведено до аналізу сильної $P$ збіжності відомих проекційних наближень $\tilde{\boldsymbol{\Lambda}}$.

Для цієї останньої неважко отримати, наприклад, оцінку

$\left\|\left(\tilde{\mathbf{\Lambda}}-\hat{\mathbf{P}}_{M} \hat{\mathbf{\Lambda}}\right) \mathbf{b}^{T}\right\| \leq\left\|\hat{\boldsymbol{\varepsilon}}_{1} \mathbf{b}^{T}\right\|+\left\|\hat{\boldsymbol{\varepsilon}}_{2} \mathbf{b}^{T}\right\|+\left\|\hat{\boldsymbol{\varepsilon}}_{3} \mathbf{b}^{T}\right\|, \quad \forall \mathbf{b} \in \ell_{2},(50)$ де введені позначення

$$
\begin{gathered}
\hat{\boldsymbol{\varepsilon}}_{1}=\hat{\mathbf{P}}_{M} \hat{\mathbf{C}}_{\varphi} \hat{\mathbf{N}}^{-1} \hat{\mathbf{M}}^{(+)} \hat{\mathbf{Q}}_{M} ; \\
\hat{\boldsymbol{\varepsilon}}_{2}=\hat{\mathbf{P}}_{M} \hat{\mathbf{C}}_{\varphi} \tilde{\mathbf{N}}^{-1} \hat{\mathbf{N}} \hat{\mathbf{Q}}_{N} \hat{\mathbf{N}}^{-1} \hat{\mathbf{M}}^{(+)} \hat{\mathbf{P}}_{M} ;
\end{gathered}
$$$$
\hat{\boldsymbol{\varepsilon}}_{3}=\hat{\mathbf{P}}_{M} \hat{\mathbf{C}}_{\varphi} \tilde{\mathbf{N}}^{-1} \hat{\mathbf{B}} \hat{\mathbf{A}}^{-1} \hat{\boldsymbol{\Theta}} \hat{\mathbf{Q}}_{M}\left[\hat{\mathbf{L}} \hat{\mathbf{A}}^{-1}+\left(\hat{\mathbf{L}} \hat{\mathbf{A}}^{-1} \hat{\mathbf{C}}_{\varphi}+2 \hat{\mathbf{C}}_{\psi}\right) \hat{\mathbf{P}}_{N} \hat{\mathbf{N}}^{-1} \hat{\mathbf{M}}^{(+)}\right] \hat{\mathbf{P}}_{M} .
$$

Цей результат означає, що необхідна збіжність безпосередньо витікає 3 фундаментальної властивості Гільбертового простору: сильній збіжності проекторів (43) до одиничного оператора, або

$$
\lim _{K \rightarrow \infty}\left\|\hat{\mathbf{Q}}_{K} \mathbf{b}^{T}\right\|=0, \quad K=M, N ; \quad \forall \mathbf{b} \in \ell_{2}
$$

Для повноти аналізу відмітимо, що проектори $\hat{\mathbf{Q}}_{M}$ та $\hat{\mathbf{Q}}_{N}$ у правій частині рівність (50) входить в різні доданки, тобто шукана збіжність матиме місце при $M, N \rightarrow \infty$ одночасно i незалежно один від одного.

\section{ЧИСЕЛЬНІ РЕЗУЛЬТАТИ: РОЗРАХУНОК ЄМНІСНОГО ШТИРЯ}

Чисельна реалізація алгоритму аналізу індуктивного штиря методом добутку областей (у загальноприйнятій постановці завдання) була виконана в роботі [10]. Тому, тут ми наводимо результати розрахунку тільки ємнісного штиря.

Елементи матриць (26), (28), (31) і (47) обчислювалися за допомогою стандартних процедур чисельної інтеграції. Виключенням $€$ оператор $\tilde{\mathbf{L}}$, для якого інтеграли виражаються через елементарні функції.

Знайдена матрична модель (45) надає можливість перевірки ряду важливих критеріїв правильності чисельного розв'язку. А саме, для 
коректно обчисленого наближення повинні виконуватися співвідношення

$$
\tilde{\mathbf{S}}^{T}=\tilde{\mathbf{S}}, \quad \hat{\mathbf{P}}-\tilde{\mathbf{S}} \hat{\mathbf{P}} \tilde{\mathbf{S}}^{\dagger}=\frac{1}{i}\left(\tilde{\mathbf{S}} \hat{\mathbf{Q}}-\hat{\mathbf{Q}} \tilde{\mathbf{S}}^{\dagger}\right)
$$

які ідентичні властивостям точного рішення. Друге 3 них $\epsilon$ закон збереження енергії в узагальненій формі [17] (тут $\hat{\mathbf{P}}$ и $\hat{\mathbf{Q}} \in$ ортопроектори на враховані хвилі, що поширюються, i на вищі типи коливань, відповідно). Далі, геометрична симетрія задачі призводить до рівностей

$$
{ }^{\alpha} \tilde{\mathbf{R}}={ }^{\beta} \tilde{\mathbf{R}}=\tilde{\mathbf{R}}\left(=\tilde{\mathbf{R}}^{T}\right) ; \quad{ }^{\alpha} \tilde{\mathbf{T}}={ }^{\beta} \tilde{\mathbf{T}}=\tilde{\mathbf{T}}
$$

Тоді наслідком (52) і (53) будуть спеціальні властивості матричних операторів

$$
\tilde{\mathbf{T}}^{T}=\tilde{\mathbf{T}}, \quad \tilde{\mathbf{V}}^{T}=\tilde{\mathbf{\Lambda}} .
$$

Характерною властивістю обчислених наближень $є$ висока міра їх збіжності (відповідні таблиці приведені в [10]), а також залежність точності виконання рівності (52) і (54) від числа типів хвиль, що враховуються. В межах варіації товщини штиря $0<r / b<0.5$ достатньо задати $M, N \geq 4$, щоб отримати необхідну для інженерних цілей точність розрахунку. За чисельний критерій коректності обчислень бралася величина норми різниці лівої і правої частини співвідношень (52) $\delta=\|\ldots\|$; співвідношення (53) завжди виконувалися (також по нормі) з точністю машинного эпсілон. Для набутих значень $M=10, \quad N=11$ проводився контроль максимального значення цієї величини.

Для числа обумовленості заданої матриці рівняння (45) введемо позначення

$$
\text { Cond }=\left\|\hat{\mathbf{P}}_{M}-\tilde{\mathbf{S}}_{0} \tilde{\mathbf{V}}\right\| \cdot\left\|\left(\hat{\mathbf{P}}_{M}-\tilde{\mathbf{S}}_{0} \tilde{\mathbf{V}}\right)^{-1}\right\| .
$$

Типова поведінка цієї характеристики показана на Рис. 2 (при обчисленнях виконувалася оцінка $\delta<4 \cdot 10^{-2}$ ). Знайдено, що для тонких штирів і поза околицями особливих точок частотного діапазону число обумовленості надзвичайне близько до одиниці, монотонно зростає зі збільшенням радіусу штиря і мало залежить від відстані $d$. При проведенні розрахунків контролювалася максимальна величина Cond .

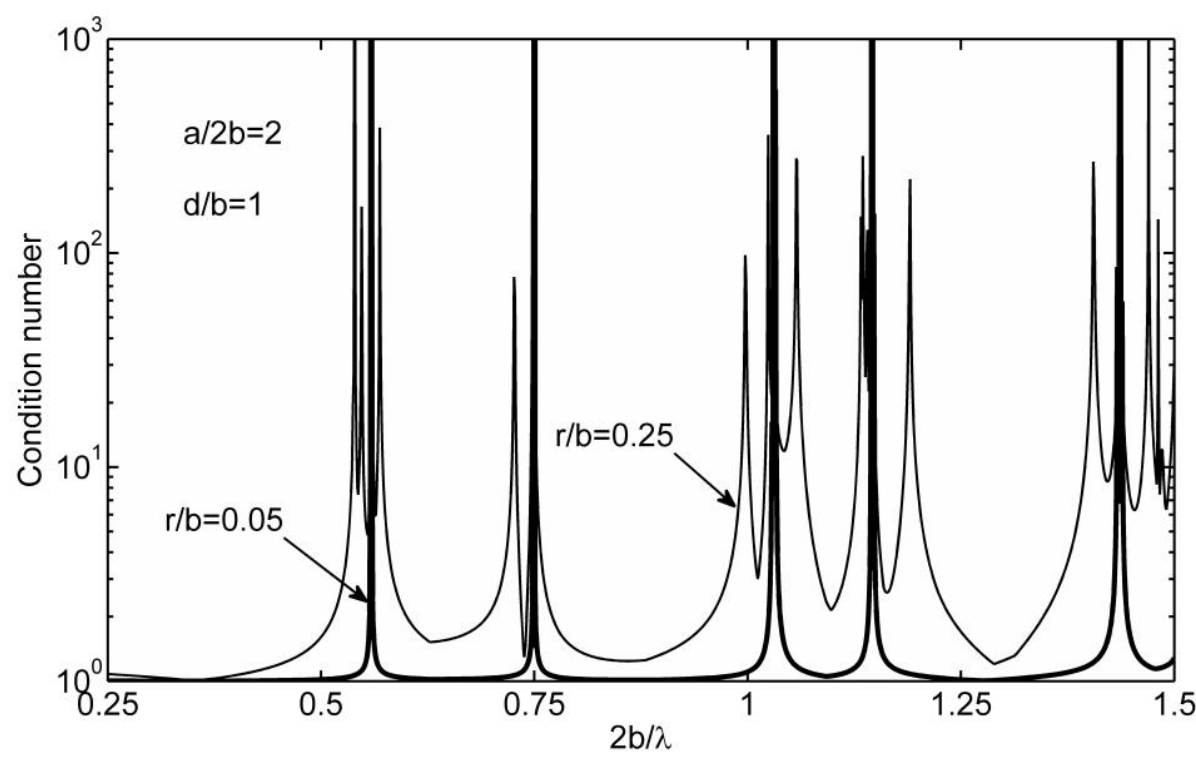

Рисунок 2 - Залежність числа обумовленості від довжини хвилі.

На Рис. 3 показана залежність модуля коефіцієнта відбиття основної моди $L E_{01}$ від товщини штиря, розміщеного на осі хвилеводу $1 \mu \times 0.6 \mu$, для хвильового числа $k=4.1 \mathrm{pa \partial /} \boldsymbol{M}$ (параметри узяті з роботи [7]).
При проведенні обчислень виконувалися нерівності $\delta<1.2 \cdot 10^{-4}, \quad$ Cond $<1.3$. Добре видно вузька область застосовності широко використовуваних на практиці апроксимацій, знайдених в роботах [2] і [3]. Дані, отримані 
методом кінцевих елементів в статті [7], добре узгоджуються з результатами розрахунку.

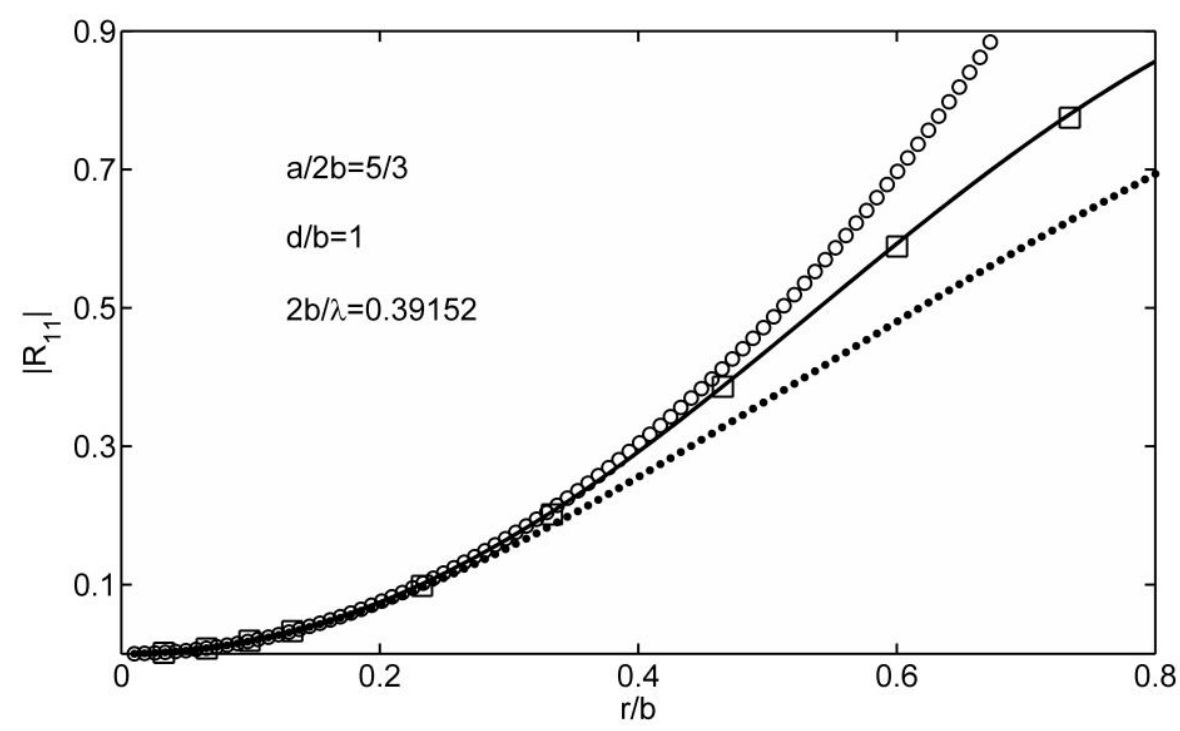

Рисунок 3 - Залежність коефіціснта відбиття від товщини штиря Суцільна лінія - МПО, кружечки ( - о - ) - розв'язки Л. Левина [3], точки ( •- ) - наближення [2], квадрати (- - - ) - дані [7].

Залежність модуля коефіцієнтів результатів розрахунку з даними роботи [8], за відображення основної моди хвилеводу від винятком випадку товстого стержня робочої довжини хвилі представлена на Рис. 4. $(r / b \geq 0.6)$ при двох і більше модах, що Для цих обчислень виконувалося $\delta<1.7 \cdot 10^{-2}$, Cond $<560$. Відмітимо хороший збіг поширюються $(2 b / \lambda>0.6)$.

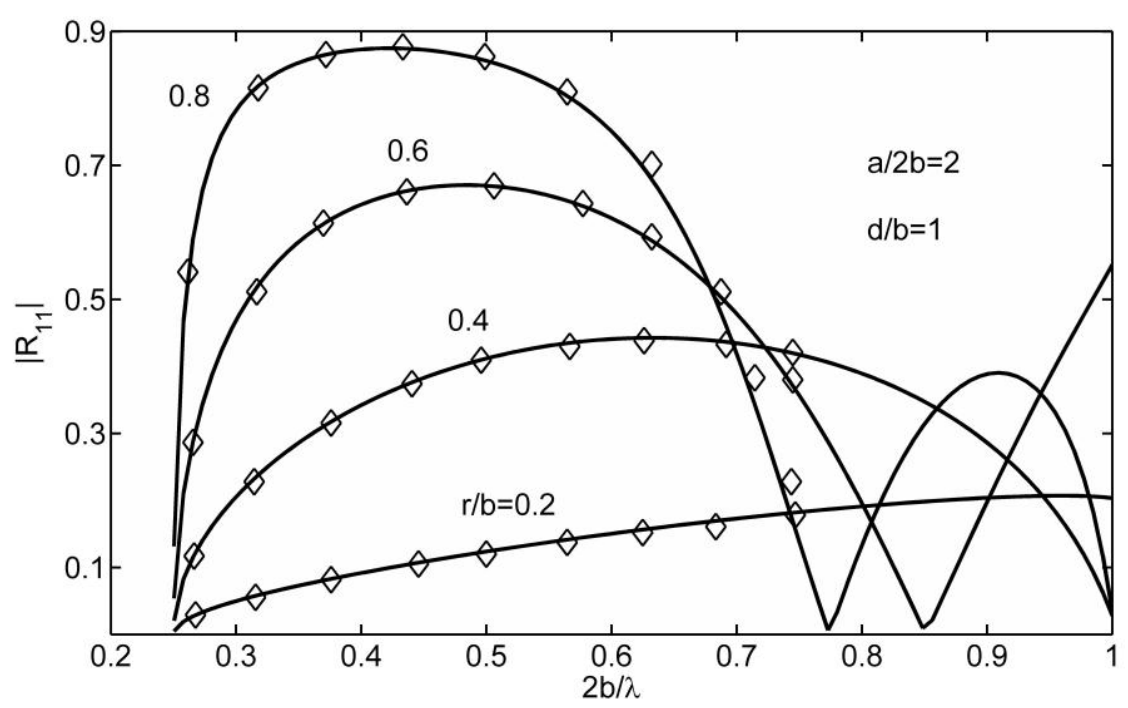

Рисунок 4 - Модуль коефіціснта відбиття як функція довжини хвилі.

Суцільна лінія - МПО, ромби ( $-\diamond-)$ - дані роботи [8].

На рис. 5 і 6 показані модуль і фаза коефіцієнта відображення хвилі $L E_{01}$ як функція відстані від центру штиря до вертикальної стінки хвилеводу. В ході обчислень виконувалося $\delta<2.3 \cdot 10^{-3}$, Cond $<1.06$. Як випливає 3 цих результатів, точність наближеного рішення, приведеного в 
довіднику [2], вище для тонких штирів, розташованих на осі хвилеводу.

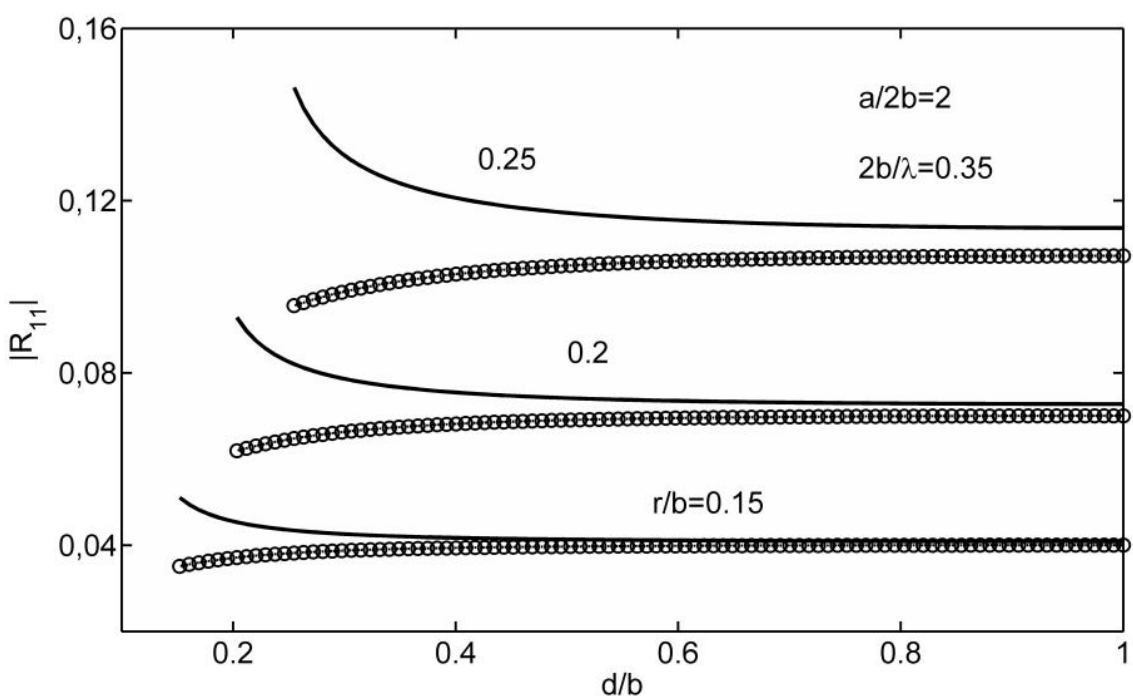

Рисунок 5 - Залежність модуля коефіцієнта відбиття від відстані між штирем і стінкою хвилеводу. Суцільна лінія - МПО, точки ( - • - ) - наближення [2].

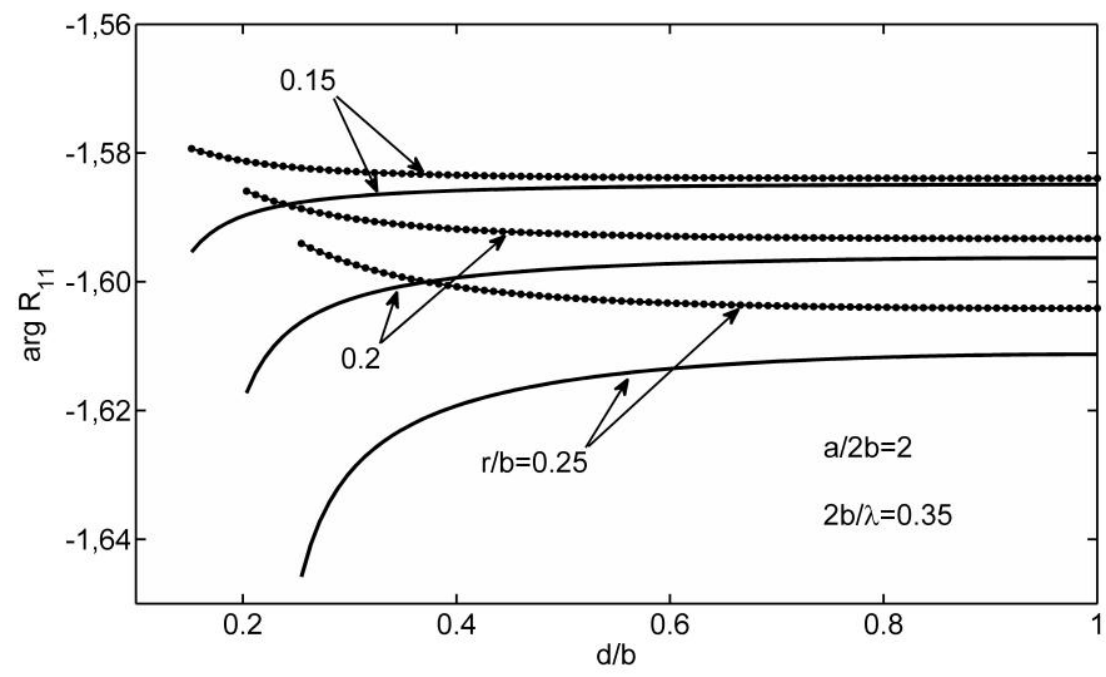

Рисунок 6 - Залежність аргументу коефіцієнта відбиття від відстані між штирем і стінкою хвилеводу. Суцільна лінія - МПО, точки ( - • - ) - наближення [2].

\section{ВИСНОВОК}

Скалярна задача розсіювання електромагнітної хвилі окремим круговим провідним циліндром, розташованим в прямокутному хвилеводі, вирішена методом добутку областей в новій постановці.

Знайдено, що шукана узагальнена матриця розсіювання $\hat{\mathbf{S}} \epsilon$ рішенням операторного рівняння, що має вигляд "правила складання тангенсів" (41). На основі відомих властивостей спектру $\sigma(\hat{\mathbf{S}})$ строго обгрунтована коректність побудованої моделі. Тим самим показано, що існування, єдиність і стійкість отриманого рішення $є$ прямим наслідком виконання закону збереження енергії.

Для цієї матричної моделі строго обгрунтовано застосування методу редукції для знаходження наближених рішень. Аналітично встановлено, що при збільшенні числа хвилеводних мод, що враховуються, має місце 
безумовна сильна $P$ - збіжність проекційних наближень до істинного рішення.

Отримана операторна модель дозволяє чисельно досліджувати характеристики розсіювання циліндричного штиря в широкому діапазоні частот при практично довільній зміні його радіусу $0<r<b$, і місця розташування в хвилеводі. Результати комп'ютерних обчислень повністю підтверджують теоретичні висновки і доводять ефективність розробленого алгоритму.

Пропонована матрична модель циліндричного розсіювача в хвилеводі може знайти застосування в САПР мікрохвильової техніки, а приведені чисельні результати слід використати в якості еталонних (тестових) даних.

Розвинений підхід може бути використаний для побудови строго обгрунтованих алгоритмів розрахунку складніших хвилеводних вузлів i пристроїв, що містять одно або декілька циліндричних включень в прямокутній області зв'язку.

1 Schwinger J. Discontinuities in waveguide / J. Schwinger, D.S. Saxon. - New York: Gordon and Breach, 1968.

2 Marcuvitz N. Waveguide Handbook / N. Marcuvitz. - N. Y.: McGraw-Hill, 1986

3 Левин Л. Теория волноводов / Л. Левин. М.: Радио и связь, 1981. - 312 c.

4 Nielsen E.D. Scattering by a cylindrical post of complex permittivity in a waveguide / E.D. Nielsen // IEEE Transactions on Microwave Theory and Techniques. - 1969. - Vol. 17, Mar.. - P. 148153.

5 Мошинский А.В. Строгое решение задачи o рассеянии волны $H_{10}$ на круговой иилиндрической неоднородности в прямоугольном волноводе/ А.В. Мошинский, В.К. Березовский // Радиотехника и электроника. 1977. - T. 22, № 7. - C. 1350-1354

6 Levitan Y. Single-post inductive obstacle in rectangular waveguide / Y. Levitan, P.G. Li, A.T. Adams, J. Perini // IEEE Transactions on Microwave Theory and Techniques. - 1983. - Vol. 31, Oct. - P. 806-811

7 Kanellopoulos V.N. A complete E-plane analysis of waveguide junctions using the finite element method / V.N. Kanellopoulos and J.P. Webb // IEEE Transactions on Microwave Theory and Techniques. - 1990. - Vol. 38, Mar. - P. 290295

8 Kishihara M. Analysis of waveguide E-plane discontinuities and components based on planarcircuit approach / M. Kishihara, T Kawai, Y Kokubo, I. Ohta // 1998 MTT-S International Microwave Symposium Digest. - Vol. 2, P. 913-916

9 Чумаченко В. П. Использование метода интегральных уравнений для решения одного класса задач электродинамики / В. П. Чумаченко // Известия вузов. Радиофизика. 1978. - T. 21, № 7. - C. $1004-1010$

10 Chumachenko V.P. Domain product technique solution for scattering by cylindrical obstacle in rectangular waveguide / V.P. Chumachenko, I.V. Petrusenko // Electromagnetics. - 2002. - Vol. 22, No. 6. - P. 473-486

11 Petrusenko I.V. Scattering by inductive post in uniformly curved rectangular waveguide / I.V. Petrusenko, V.P. Chumachenko // IEE Proceedings. - Microwaves, Antennas and Propagation. - 2003. - Vol. 150, No. 6. - P. 498-50

12 Petrusenko I.V. Analytic-numerical analysis of waveguide bends / I. V. Petrusenko // Electromagnetics. - 2004. - Vol. 24, No. 4. - P. 237-254

13 Petrusenko I.V. Matrix operator technique for analysis of wave transformers / I.V. Petrusenko // 2004 MMET'04 Proceedings of the $10^{\text {th }}$ International Conference on Mathematical Methods in Electromagnetism. - P. 118-120

14 Petrusenko I.V. Fresnel formulae for scattering operators / I.V. Petrusenko, Yu. K. Sirenko // Telecommunications and Radio Engineering. - 2011. - V. 70. - P. 749-758.

15 Вейль Г. Классические группь: их инварианты и представления / Г. Вейль. - М.: Гос. издательство иностранной литературы, 1947. $-408 c$

16 Petrusenko I.V. Generalization of the power conservation law for scalar mode-diffraction problems / I.V. Petrusenko, Yu. K. Sirenko // Telecommunications and Radio Engineering. 2009. - V. 68. - P. 1399-1410

\section{Поступила в редакцію 02.12.2018 р.}

Рекомендували до друку: докт.техн.наук, проф. Олійник А. П., докт. техн. наук, проф. Горбійчук М. I. 